\title{
Das Elterngeld: Wahlfreiheit und Existenzsicherung für (alle) Eltern?
}

Das 2007 eingeführte Elterngeld stellt eine der wichtigsten familienpolitischen Neuerungen der letzten Jahre dar. Es markiert zum einen eine Zäsur in den bisherigen Orientierungen in der Familienpolitik und rückt zum anderen die Begriffe Wahlfreiheit und Autonomie in den Mittelpunkt familienpolitischer Verhandlungen. Diese Begriffe werden in der öffentlichen Debatte unterschiedlich gedeutet. Aus sozialwissenschaftlicher Perspektive stellt sich die Frage, ob das Elterngeld als familienpolitisches Instrument tatsächlich Wahlfreiheit oder Autonomie ermöglicht und unter welchen Bedingungen dies der Fall ist. Empirisch gilt es zu klären, inwiefern sich aus dem Umfang seiner Nutzung ein Hinweis auf Wahlfreiheit und ihre Inanspruchnahme ableiten lässt.

SABINA SCHUTTER, CLAUDIA ZERLE-ELSÄßER

\section{Wahlfreiheit unter dem „Verdikt der Employability“}

Die von Beck (1986) unter dem Stichwort „Individualisierung" beschriebene Herauslösung des Individuums aus gesellschaftlichen Zusammenhängen und die Abnahme der Verbindlichkeit normativer Leitbilder für Lebensentwürfe stellen den Einzelnen vor eine Vielzahl biografischer Entscheidungen, deren Wahl von verschiedenen Parametern beeinflusst wird. Sind es nicht mehr Schicht oder wirtschaftliche Zwänge (z. B. Kinder als Status, als Erben oder als Arbeitskraft), die zu einer Entscheidung für Kinder führen, bilden Kinder heute eher einen Teil eines individuellen Lebensentwurfs, an den Glücks- und Verwirklichungserwartungen geknüpft werden. Hinzu kommen neue Freiheiten beim Zusammenfinden von Partnerschaften, die zunehmend das Leben vervollständigen und zu individueller Erfüllung führen sollen: „In Idealisierungen des modernen Liebesideals spiegelt sich noch einmal der Weg der Moderne. Die Überhöhung ist das Gegenbild zu den Verlusten, die diese hinterlässt. Gott nicht, Priester nicht, Klasse nicht, Nachbar nicht, dann wenigstens Du. Und die Größe des Du ist die umgedrehte Leere, die sonst herrscht." (Beck 1986, S. 187f.). Das bringt mit sich, dass Partnerschaften heute nicht mehr aus gesellschaftlichen Zwängen aufrechterhalten werden, wenngleich die heterosexuelle Paarbeziehung noch immer die dominante Lebensform erwachsener Menschen ist. Im Hinblick auf Familiengründung heißt das, dass eine Familie nicht mehr selbstverständlicher Teil der Biografien und Wünsche junger Erwachsener ist. Dieser scheinbare Zugewinn an Handlungsmöglichkeiten durch die geringere Bindungskraft normativer Zwänge kehrt im Rahmen wirtschaftspolitischer Erwägungen wieder, nämlich in Gestalt der Frage, ob sich Paare Kinder „leisten“ können. Im Zusammenspiel mit den Veränderungen der Erwerbsarbeit stellt sich dies wie folgt dar:

Das „Verdikt der Employability“ (Jurczyk/Schier et al. 2009) beinhaltet, dass spätestens im Nachgang der Lissabon-Strategie die Steigerung der Frauenerwerbstätigkeit erklärtes Ziel aller EU-Staaten ist, mithin auch in Deutschland. Dies deutet sich z. B. auch in der Reform des Unterhaltsrechts an, das eine massive Einschränkung des nachehelichen Unterhalts für (in der Regel) Ehefrauen und des Betreuungsunterhalts für nicht verheiratete Elternteile mit sich bringt und grundsätzlich von einer vollen Erwerbstätigkeit ausgeht, sobald das betreute Kind drei Jahre alt ist. Eine Einschränkung eigener Erwerbstätigkeit über dieses Alter des Kindes hinaus muss entweder in einem erhöhten Betreuungsbedarf des Kindes begründet sein oder sie findet auf eigenes Risiko statt.

Das Elterngeld, als Lohnersatzleistung im ersten Jahr nach der Geburt eines Kindes konzipiert, enthält ähnliche Strukturen, da es sich an der Norm erwerbstätiger Eltern orientiert. Die Gesetzesbegründung enthält den Hinweis, dass das Elterngeld „Erwerbsanreize“ setzen (BT-Drs. 
16/1889, S. 23) und entgegen dem Erziehungsgeld nicht mehr nur allein die Betreuungsleistung finanziell unterstützen solle.

Aus der Perspektive des Geschlechterverhältnisses sind Sorgeaufgaben und Haushaltstätigkeiten sowie Erwerbstätigkeit ungleich verteilt. Die Daten des DJI-Surveys „Aufwachsen in Deutschland: Alltagswelten“ (AID:A) belegen, dass ein überwiegender Anteil von Paaren mit Kindern im Haushalt das traditionelle oder modernisierte Ernährermodell lebt. Dies gilt auch weit über das dritte Lebensjahr des Kindes hinaus (vgl. Wirth/Schutter 2011). Dennoch nehmen die Erwerbswünsche von Frauen zu und auch die Anteile von Müttern, die zumindest in Teilzeit erwerbstätig sind, befinden sich im aufsteigenden Trend. ${ }^{\bullet}$

Familiengründungen sind also nicht das Ergebnis gesellschaftlicher Normen, sondern sie sind das Resultat von Lebensentwürfen und biografischen Entscheidungen, denen individuelle Glücksvorstellungen zugrunde liegen. Die Frauenerwerbstätigkeit ist angestiegen, wobei der Ausbau der Kinderbetreuung dem Ausbau der Erwerbsbeteiligung noch hinterherhinkt. Gleichzeitig ist die Frauenerwerbsbeteiligung, insbesondere die der Mütter, auch explizit politisches Ziel. In diesem Kontext stellt das Elterngeld eine Maßnahme dar, die sowohl den Entscheidungsprozess zur Familiengründung als auch die Entscheidung für oder gegen Erwerbstätigkeit beeinflussen soll. Im Folgenden soll untersucht werden, wie sich das Elterngeld auf Entscheidungsprozesse von Erwachsenen auswirkt. Bothfeld (2005) hat für den Übergang vom Erziehungsurlaub zur Elternzeit festgestellt, dass es sich hierbei um die Etablierung der Idee der Absicherung durch eigene Erwerbstätigkeit handelt. Für das Verhältnis von Wahlfreiheit und Autonomie hält sie fest, dass es sich bei Ersterem um ein politisches Leitbild handelt, das jedoch nur unter den Bedingungen der tatsächlichen Autonomie von Entscheidungen funktionieren kann. Dies würde voraussetzen, dass sowohl materielle Einbußen wie auch ideelle Leitbilder autonome Entscheidungen ermöglichen (vgl. Fraser 1994). Saxonberg (2009) geht davon aus, dass eine gleichstellungsorientierte Familienpolitik sowohl für traditionelle Familientypen als auch für gleichberechtigte und für alternative Familienformen den höchsten Spielraum der Wahlfreiheit ermöglicht.

Im Hinblick auf die Wirkung des Instruments „Elterngeld" können vor allem drei Optionen als regelhaft angenommen werden: zum einen die grundsätzliche Entscheidung für oder gegen Elternschaft, zum anderen das ob und wie der Reduktion/des Ausstiegs aus der Erwerbstätigkeit und zum dritten die Frage, wer von zwei Eltern, wann und wie lange, die Erwerbstätigkeit unterbricht oder reduziert. Diese Entscheidungen werden vor dem Hintergrund bestimmter sozialpolitischer Rahmenbedingungen getroffen, von denen das Elterngeld eine darstellt.

Zwei Fragen sind für diesen Beitrag zentral: Wie steht das Elterngeld im Verhältnis zu diesen Daten und leitenden Annahmen? Wird durch das Elterngeld eine Wahlfreiheit für alle Familien ermöglicht?
Doch zunächst soll beleuchtet werden, wie die Begriffe Wahlfreiheit und Autonomie in der öffentlichen Debatte um das Elterngeld verhandelt wurden und werden.

\subsection{Wahlfreiheit und Autonomie in der öffentlichen Debatte}

Die oben entwickelten drei Entscheidungsdimensionen lassen sich in der öffentlichen Debatte zur Einführung des Elterngeldes und zur Wahlfreiheit identifizieren.

Im Rahmen einer bevölkerungsorientierten Familienpolitik sollte das Elterngeld als Lohnersatzleistung erstens auch dazu dienen, die Geburtenzahlen zu erhöhen, insbesondere bei den Frauen, die als Verantwortliche des Geburtenrückganges identifiziert wurden: kinderlose Akademikerinnen (vgl. kritisch Auth 2007; Schutter 2005; als Beleg BMFSFJ 2005). Verbunden mit einem nicht unerheblichen Ausmaß an „Alarmismus“ (Auth 2007) stand im Zentrum der Debatte, dass junge Akademikerinnen zu wenige Kinder gebären würden und daher „die Falschen “ ${ }^{\text {(2) }}$ die Kinder bekämen, die „sozial Schwachen“.

Durch die einkommensabhängige Gestaltung des Elterngeldes (67 \% ihres Nettogehaltes für zwölf Monate) erhalten Eltern mit höheren Einkommen mehr Lohnersatz bei Unterbrechung der Erwerbstätigkeit und dies, so die Annahme bei Einführung des Elterngeldes, würde besser verdienenden (jungen) Frauen die Entscheidung für ein Kind erleichtern. Seit 2002, mit der Übernahme des Familienministeriums durch Renate Schmidt (SPD), wurde das Thema Bevölkerungsorientierung wieder zu einer Zielsetzung der Familienpolitik. Die entsprechenden Politikmaßnahmen wurden in der Folge von Ursula von der Leyen (CDU) weitergeführt (vgl. z. B. Auth 2007): „Es handelt sich um eine Maßnahme [...], um die Opportunitätskosten von Kindern zu senken, vor allem für gut qualifizierte, gut verdienende Frauen bzw. Familien.“ (ebd., S. 96).

In diesem Sinne stellte das Elterngeld eine politische Zäsur dar, der eine ideologische Neuorientierung der Familienpolitik vorausging (eindrücklich hierzu: BMFSFJ 2005).

In der zweiten Dimension, der Wahl und Dauer einer Erwerbsunterbrechung, beinhaltete die Begrenzung des Elterngeldes auf maximal zwölf Monate für einen Elternteil (und zwei zusätzliche Partnermonate) gegenüber der zuvor geltenden Erziehungszeit von drei Jahren, wobei zwei

Hierbei sei erwähnt, dass die Arbeitsmarktbedingungen, die für eine Teilzeittätigkeit bzw. eine gering entlohnte Arbeit von Müttern sprechen, wie z. B. die Mitversicherung von Ehepartnern, in diesem Artikel zwar nicht beleuchtet werden, gleichwohl einen massiven Anreiz zur Teilzeitarbeit von Müttern darstellen.

(2) So der heutige Bundesgesundheitsminister Daniel Bahr in der Bild am Sonntag, 23. 01. 2005, zitiert nach tagesschau. de, http://www.tagesschau.de/inland/meldung202404.html. 
Jahre lang der Bezug des Bundeserziehungsgeldes finanzielle Unterstützung bot, eine deutliche Verkürzung. Alternativoptionen wie die Halbierung des Elterngeldes weichten diese Eingrenzung zwar auf, grundsätzlich lässt sich jedoch festhalten, dass ein zwölfmonatiger Zeitraum im Mittelpunkt stand.

Die dritte Entscheidungsdimension, die Verteilung der Elternzeit zwischen zwei Eltern, wurde durch die Option der zwei Partnermonate beeinflusst. Indem zwei der vierzehn Monate Elterngeld vom jeweils anderen Partner bezogen werden sollten, standen explizit Väter im Mittelpunkt der politischen Zielsetzung. „Die Regelung soll insbesondere Vätern die Möglichkeit eröffnen, eine aktivere Rolle in der Familie zu übernehmen, und ihnen auch gegenüber Dritten die Entscheidung erleichtern, sich eine Zeit lang der Betreuung ihres neugeborenen Kindes zu widmen." (BTDrs. 16/1889, S. 16). Diese Bindung von mindestens zwei Monaten an den Partner sollte die Anteile von (erwerbstätigen) Vätern, die Elternzeit beanspruchen, erhöhen.

\subsection{Wahlfreiheiten und ihre Deutungen}

Mit dem Begriff der Wahlfreiheit waren in der öffentlichen Debatte mitunter einander widersprechende Ziele verbunden. Für die Gegner des Elterngeldes stellte die Maßnahme gegenüber dem bisherigen Erziehungsgeld eine Einschränkung der Handlungsmöglichkeiten dar. Auch die Bindung von zwei Monaten Elterngeldbezug an die Beteiligung des Partners (Adressaten der Maßnahme waren vor allem die Väter) wurde hämisch als „Wickelvolontariat“ und damit ebenso als Einschränkung elterlicher Handlungsspielräume bezeichnet. ${ }^{3}$ Seitens derer, die eine möglichst lange Betreuung von Kindern durch ihre Eltern befürworten, war mit Wahlfreiheit vor allem eine lange finanzielle Absicherung von beruflichen Auszeiten (meist der Mütter) gemeint. ${ }^{\circledR}$

Mit Wahlfreiheit waren seitens der Befürworter des Elterngeldes, die u. a. kurze Erwerbsausstiege von Müttern befördern wollten, eine schnelle Rückkehroption und die damit verbundenen Vorteile im Erwerbsleben sowie für die wirtschaftliche Absicherung gemeint. Das Elterngeld sollte Erwerbsanreize setzen: dies zum einen durch die einkommensabhängige Gestaltung, zum anderen durch die Beschränkung auf ein Jahr Bezugszeitraum. Das bedeutet, dass familienpolitisch widerstreitende Leitbilder zur Wahlfreiheit existierten und bis in die Gegenwart bestehen (vgl. Jurczyk/Schutter 2012).

\subsection{Elterngeld und Arbeitsteilung}

Bezogen auf das Elterngeld kommt die Komponente einer Neuverteilung von familialen Aufgaben zwischen den Geschlechtern hinzu. Die sogenannten Partnermonate sollen gewährleisten, dass auch Väter ihre Erwerbstätigkeit begrenzt unterbrechen können, damit die stark auf Mütter fokussierte Übernahme von care (Sorgearbeit) durchbrochen wird. Dies soll sowohl Vätern ermöglichen, auch fa- miliale Aufgaben zu übernehmen, wie für Mütter Freiheiten schaffen, früher in die Erwerbstätigkeit zurückzukehren. Allerdings werden die zwei zusätzlichen Monate nur Partnern gewährt, die ihre Erwerbstätigkeit unterbrechen. Nicht erwerbstätige Partner bekommen keine zusätzliche Elterngeldleistung. Das heißt, die Partnermonate werden nur dann gewährt, wenn mindestens ein Partner vor der Elternzeit erwerbstätig war, und sie richten sich vor allem an erwerbstätige Väter. Im Sinne von Connells (2006) Konzept der Binnenbeziehungen von Geschlecht wurde quasi eine neue Form untergeordneter Vaterschaft geschaffen: Väter, die nicht erwerbstätig sind und anscheinend nicht in die Väterlichkeit involviert werden sollen. ${ }^{\ominus}$ Die Leitbilder, dass ein Kind im ersten Jahr nach der Geburt bei den Eltern zu Hause den Hauptteil seiner Zeit verbringen sollte, das Leitbild des involvierten Vaters, die Leitbilder von guter Mutterschaft und gleichzeitig das der erwerbstätigen, gut verdienenden Mutter stehen einerseits im Widerstreit, andererseits ergänzen sie sich.

\subsection{Elterngeld und Familien in prekären Lebenslagen}

Über diese teilweise polemische Debatte geriet vor allem die Gruppe derjenigen aus dem Blick, die weder von $67 \%$ ihres Nettogehaltes ihre Existenz sichern können, weil sie weder genug Einkommen haben, noch die realistische Aussicht, nach zwölf Monaten wieder in einem Beruf zu arbeiten, z. B. weil es an Kinderbetreuung mangelt, weil sie in nicht abgesicherter geringfügiger Beschäftigung oder in Kleinstbetrieben ohne Kündigungsschutz gearbeitet haben. Ein weiterer Aspekt blieb unbeleuchtet: Mit der Einführung des Elterngeldes war der Ausbau der Kindertagesbetreuung mit dem Rechtsanspruch ab einem Jahr noch lange nicht gegeben. Dieser wird erst mit dem Inkrafttreten des Kinderförderungsgesetzes (KiFöG 2008) Gesetzeslage und,

3 Z. B. Peter Ramsauer (CSU) in der Berliner Zeitung vom 26. 04. 2006, http://www. berlinonline.de/berliner-zeitung/archiv/.bin/dump.fcgi/2006/0426/politik/0017/ index.html.

(4) Interessant daran ist, dass sich unterschiedliche Begründungen dieses Lebensmodells finden lassen, die mitunter auch mit kindlichen Bedürfnissen begründet werden. Dies kann hier nicht Gegenstand der Untersuchung sein (vgl. dazu Bühler-Niederberger 2005; Schutter 2011).

5 Grundsätzlich ist es nicht selbstverständlich, dass Väter, auch wenn sie nicht erwerbstätig sind, nach der Geburt eines Kindes Fürsorgeaufgaben übernehmen. Das Elterngeld kann einerseits als Anreiz verstanden werden, dass Väter ihre Erwerbstätigkeit unterbrechen, es kann aber auch als Anreiz für Väter betrachtet werden, sich stärker an Familienaufgaben zu beteiligen. Es stellt sich vor diesem Hintergrund die Frage, ob es für Väter ohne Erwerbstätigkeit keinen Anreiz geben soll, sich an den Familienaufgaben zu beteiligen. 
wenn überhaupt, im Jahr 2013 Realität. Das heißt, mit Inkrafttreten des Elterngeldes im Jahr 2007 war die Betreuung für unter Dreijährige noch nicht in einem Ausmaß gesichert, dass diejenigen Eltern, die in den Jahren Jahr 2007 bis 2013 Elternzeit nehmen, nach zwölf Monaten über eine gesicherte Kinderbetreuung verfügen.

\section{Familienpolitische Geldleistung im Wandel}

\subsection{Vom Erziehungsgeld zum Elterngeld}

Das Elterngeld stellt eine familienpolitische Zäsur dar, da es den Paradigmenwechsel der Familienpolitik von einer sozialpolitischen Ausrichtung hin zu einer auch bevölkerungsorientierten Zielsetzung markiert. Die Veränderungen gegenüber dem zuvor wirksamen Erziehungsgeld stellten sich wie folgt dar (Übersicht 1): Das Erziehungsgeld in Höhe von $300 €$ wurde für zwei Jahre gezahlt und nicht auf weitere Sozialleistungen angerechnet. Die Budgetvariante von $450 €$ wurde ein Jahr ausgezahlt. Beiden Leistungen war gemeinsam, dass das Höchsteinkommen beider Partner bei $30.000 €$ (netto) in den ersten sechs Lebensmonaten des Kindes und bei $16.500 € \mathrm{ab}$ dem siebten Lebensmonat des Kindes lag und über diesem Betrag kein Erziehungsgeld gezahlt wurde (für Alleinerziehende: $19.500 €$ ). Die Erwerbstätigkeit durfte maximal 30 Stunden pro Woche betragen.

Beim Elterngeld werden (abgesehen von Ausnahmen) $67 \%$ des durchschnittlichen, durch den vorübergehenden Erwerbsausstieg entfallenden Nettoeinkommens im Jahr vor der Geburt ersetzt. Der Mindestbetrag liegt bei $300 €$, der Höchstbetrag bei $1.800 €$. Geringverdiener mit einem Nettoeinkommen unter $1.000 €$ erhalten pro $20 €$, die ihr Einkommen unter $1.000 €$ liegt, ein Prozent mehr Lohnersatz. Zusätzlich enthielt das Elterngeld ursprünglich sozialpolitische Komponenten, denn die $300 €$ Mindestelterngeld waren zunächst nicht an die Voraussetzung der Erwerbstätigkeit gebunden. Die Bedingung für den Bezug von zwei zusätzlichen Partnermonaten ist die Erwerbstätigkeit beider Partner. Elternpaare, die erwerbslos waren, erhalten grundsätzlich nur zwölf Monate Elterngeld (vgl. Bundeselterngeld- und Elternzeitgesetz [BEEG] $\$ 4$ Abs. 3). Alleinerziehende können allein 14 Monate Elterngeld beziehen, wenn sie das alleinige Sorgerecht haben und vor der Geburt des Kindes erwerbstätig waren.

Beim Elterngeld handelt es sich um eine Lohnersatzleistung mit einer sozialen Komponente. In einer Pressemitteilung des Bundesfamilienministeriums wurde zur Einführung des Elterngeldes festgestellt: „Das Elterngeld ist im Gegensatz zu Sozialhilfe oder Arbeitslosengeld II keine Sozialleistung des Staates. Es steht für einen Perspektivwechsel in der Familienpolitik.“ (BMFSFJ 2006).
ÜBERSICHT 1

Gegenüberstellung von Bundeserziehungsgeld und Elterngeld in alter und neuer Fassung

\begin{tabular}{lllll}
\hline & Erziehungsgeld & Elterngeld & Elterngeld ab 01.01.2011 \\
\hline Dauer & 24 Monate & $12+2$ Monate & $12+2$ Monate \\
\hline Alternativ-Dauer & 6 Monate & 28 Monate & 28 Monate \\
\hline Mindesthöhe & $300 €$ & $300 €$ & $300 €$ \\
\hline Höchstgrenze & $450 €$ & $1.800 €$ & $1.800 €$ \\
\hline Lohnersatz & nein & $67 \%$ & $67 \%-65 \%$ (nach Einkommenshöhe) \\
\hline Ausschlusskriterien & $\begin{array}{l}\text { Ab 7. Monat } \\
\text { Einkommenshöhe }\end{array}$ & $\begin{array}{l}\text { Bestimmte Gruppen } \\
\text { von Migranten }\end{array}$ & $\begin{array}{l}\text { Bestimmte Gruppen von Migranten und } \\
\text { Beziehern von SGB-II-Leistungen, wenn } \\
\text { vor der Geburt keine Erwerbstätigkeit } \\
\text { bestand. }\end{array}$ \\
\hline
\end{tabular}

Quelle: Zusammenstellung der Autorinnen

\subsection{Elterngeld im Sparpaket}

Mit dem „Sparpaket“ der Bundesregierung hat das Elterngeld zum 1. Januar 2011 Veränderungen erfahren (Übersicht 1). Der Mindestbetrag des Elterngeldes wird nun auf Leistungen nach dem SGB II angerechnet, wenn die Eltern nicht vor der Elternzeit erwerbstätig waren. Für Eltern im SGB-II-Bezug, die zuvor erwerbstätig waren und Elterngeld beziehen, wird ein „Elterngeldfreibetrag“ eingerichtet. Ab einer Grenze von $1.200 €$ Nettoeinkommen werden statt $67 \%$ nur noch $65 \%$ des Nettoeinkommens ersetzt. Der Mindestbetrag wird weiterhin an Studierende, Hausfrauen oder anderweitig nicht Erwerbstätige gezahlt. Lediglich Bezieher von SGB-II-Leistungen erhalten die Anrechnung. Die ursprüngliche Zielgruppe des Erziehungsgeldes bleibt weitgehend von der Leistung ausgeschlossen (vgl. Änderungen des Bundeselterngeldes und Elternzeitgesetzes BEEG durch Art. 14 des Gesetzes vom 09. 12. 2010).

\section{Empirische Befunde}

\subsection{Daten zur Nutzung des Elterngeldes}

Zur Nutzung des Elterngeldes liegen aus der amtlichen Elterngeldstatistik zahlreiche Informationen vor, wie etwa zum Geschlecht der Nutzenden, der Dauer der Nutzung, dem Timing der Bezugszeit und der Höhe des ausbezahlten Elterngeldes. Die Erfassung der Daten erfolgt, deutschlandweit sowie auf Länderebene, quartalsweise über die als beendet gemeldeten Leistungsbezüge. Die aktuellsten vorliegenden Daten beziehen sich auf das 3. Quartal 2011, in dem in ganz Deutschland 225.114 Elterngeldbezüge beendet wurden (Statistisches Bundesamt 2011). Diese Daten 
erlauben uns Aussagen über den Umgang der Nutzerinnen und Nutzer mit dem Elterngeld.

Nachvollziehen lässt sich mit den Daten daher, wer von beiden Elternteilen heute, mehr als vier Jahre nach der Einführung des Instrumentes Elterngeld, wann und wie lange die Erwerbstätigkeit unterbricht oder reduziert. Weiterhin gestatten die Angaben zur Bezugshöhe des Elterngeldes Aussagen darüber, für welche Elterngeldbezieher das Elterngeld, wie ursprünglich anvisiert, überhaupt existenzsichernd ist.

Nicht abbilden lässt sich jedoch mit diesen Daten, inwieweit das Elterngeld die Entscheidung für oder gegen Elternschaft beeinflusst und welcher Anteil der Eltern nach Ablauf des Elterngeldes weiterhin (unbezahlte) Elternzeit nimmt, weil die Betreuung des Kindes institutionell nicht abgesichert ist.

\subsection{Geschlechtsspezifische Nutzung des Elterngeldes}

Wie aus der Elterngeldstatistik hervorgeht, bezogen im 3. Quartal 2011 172.377 Mütter (76,6 \%) und 52.737 (23,4 \%) Väter Elterngeld. Die Nutzung des Elterngeldes nach der Ge-

ABB. 1

\section{Entwicklung des Väteranteils an allen beendeten Leistungsbezügen \\ Angaben in Prozent}

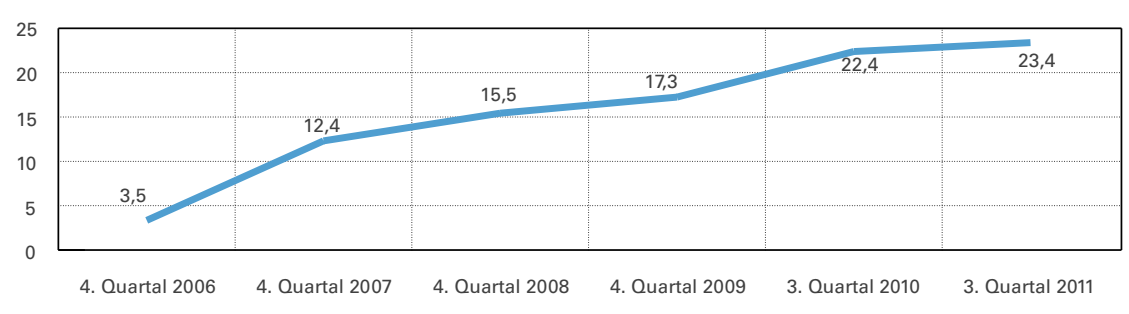

Quelle: Statistisches Bundesamt 2011

\section{TABELLE 1}

Dauer der Inanspruchnahme von Elterngeldmonaten von Müttern und Vätern Anteile in Prozent

\begin{tabular}{lc|c|c|c|c}
\hline & $\begin{array}{c}\text { Bis zu 2 } \\
\text { Monate }\end{array}$ & $\begin{array}{c}\text { 3 bis 9 } \\
\text { Monate }\end{array}$ & $\begin{array}{c}\mathbf{1 0} \text { bis 12 } \\
\text { Monate }\end{array}$ & $\begin{array}{c}\mathbf{1 3} \text { bis 14 } \\
\text { Monate }\end{array}$ & Durchschnitt \\
\hline Mütter & 0,8 & 4,5 & 92,6 & 2,0 & 11,6 \\
\hline Väter & 78,5 & 13,6 & 7,9 & 0,0 & 3,2 \\
\hline
\end{tabular}

burt eines Kindes hat damit im Vergleich zum Erziehungsgeld bei den Vätern deutlich an Bedeutung gewonnen. Waren es Ende 2006 noch gerade 3,5\% der Väter, die in Erziehungszeit gegangen sind, sind heute $23,4 \%$ der Elterngeldbezieher Väter (Abbildung 1).

Wenngleich dies ein enormer Anstieg war, geht aus diesen Zahlen auch hervor, dass die Übernahme von Elternmonaten für die Väter bislang nur in einer Minderheit der Familien möglich zu sein scheint: In 75,7 \% der Familien, in denen die Mütter Elterngeld in Anspruch nahmen, hat dies der zugehörige Vater nicht getan. ${ }^{\odot}$ In einem Großteil der Familien bleiben die Frauen also die Hauptverantwortlichen, in nur 6,1 \% der Familien übernehmen alleine die Väter Elternmonate. In den meisten Fällen (93,5\%) nehmen die Väter ihre Partnermonate zusätzlich zu den Müttern, stellen also Partneranträge. Mit zwei Partnermonaten übernimmt der Großteil der Väter (78,5 \%) zudem den deutlich kürzeren Teil der insgesamt 14-monatigen Elterngeldphase. So beträgt die Bezugsdauer des Elterngeldes bei den Vätern im Durchschnitt nur 3,2 Monate, während es bei den Müttern, von denen 92,6 \% zwischen zehn und zwölf Monaten nehmen, im Schnitt 11,6 Monate sind, die sie zu Hause bleiben oder ihre Arbeitszeit reduzieren (Tabelle 1).

Die meisten Väter legen ihre Partnermonate an den Beginn (29,6 \%) oder an das Ende (17,4 \%) der Elterngeldphase. Wie die Studie von $P f a h l / R e u ß$ zum neuen Elterngeld darüber hinaus zeigt, nimmt die Hälfte der Väter die Partnermonate parallel und nicht zeitlich versetzt zur Mutter des Kindes (Pfahl/Reuyß 2009, S. 58). Die jungen Väter wünschen sich gerade in den frühen Phasen der Familienbiografie insgesamt mehr gemeinsame Familienzeit, welche die Herstellung von Familie (vgl. Jurczyk/Keddi et al. 2009) möglich macht. Ihre Motive zur Nutzung von Vätermonaten beziehen sich daher vorwiegend auf die Familie als Ganzes (Pfahl/Reuyß 2009, S. 73).

Trotz genderneutraler Gesetzesformulierung ist der Bezug von Elterngeld, so zeigen die vorliegenden Daten, deutlich geschlechtsspezifisch ausgestaltet. Mütter beziehen deutlich häufiger und wesentlich länger Elterngeld als es die Väter tun. Mögliche Gründe dafür gehen zwar nicht aus der Elterngeldstatistik hervor, wurden jedoch im Rahmen der Evaluation von Nutzung und Wirkungen des neuen Elterngeldgesetzes behandelt.

\subsection{Gründe für die Nichtbeanspruchung des Elterngeldes von Vätern}

Aus der Evaluationsstudie des Rheinisch-Westfälischen Instituts für Wirtschaftsforschung (RWI) geht hervor, dass in $68 \%$ der Familien, in denen keine Vätermonate übernom-

(6) In diesen Familien wurden keine Partneranträge gestellt. Diese Zahl ist extra ausgewiesen, unterscheidet sich daher geringfügig von dem Anteil an Müttern bzw. Vätern an allen Anträgen. 
men wurden, „eine Reduktion der Arbeitszeit nicht möglich war“ (RWI 2009, S. 16). Ein Drittel der Familien (35 \%) nannte berufliche/betriebliche Gründe als Hauptfaktor, $45 \%$ sahen in finanziellen Gründen ein Haupthindernis (ebd.). Auf das (volle) Einkommen des Vaters konnte nicht verzichtet werden. In nur $20 \%$ der befragten Familien ohne väterlichen Elterngeldbezug steckte hinter der traditionellen Aufteilung auch ein explizit traditionelles Familienbild, demzufolge sich besser „die Partnerin ganz um das Kind kümmern“ (ebd.) soll.

\subsubsection{Betreuen Väter ältere Kinder?}

Unklar bleibt die Bedeutung, die dem Alter des Kindes für die Übernahme von Betreuungszeiten des Vaters zukommt. Während einige Befunde darauf hindeuten, dass sich das Engagement der Väter grundsätzlich kaum verstärkt, je älter das Kind wird, weisen andere Befunde darauf hin, dass Bedeutung und Betreuungsanteil des Vaters wachsen. Fthenakis/Minsel (2002, S. 152) etwa konnten in ihren Analysen keinen Anstieg der Väterbeteiligung zwischen dem 6. und dem 20. Lebensmonat des Kindes beobachten. Der RWI-Studie (2009, S. 24) zufolge steigt der Betreuungsanteil der Väter insgesamt mit dem Alter des Kindes ein wenig an: von $11 \%$ im ersten Lebensjahr des Kindes auf $16 \%$ im zweiten. Deutlicher wird der Anstieg der Väterbeteiligung jedoch, wenn man speziell die Väter betrachtet, die auch Elterngeldmonate übernehmen: Im ersten Lebensjahr des Kindes übernehmen die Väter in Familien ohne Partnerantrag im Schnitt $8 \%$ der gesamten Betreuungszeit, jedoch $23 \%$ in Familien mit Partnerantrag. Im zweiten Jahr übernehmen Väter in Familien ohne Partnerantrag $12 \%$ und in Familien mit Partnerantrag $31 \%$. Noch höher liegt der väterliche Betreuungsanteil im zweiten Lebensjahr des Kindes, wenn die Mutter nach zwölf Monaten wieder eine Erwerbstätigkeit aufnimmt.

\subsubsection{Hegemoniale Mütterlichkeit}

Ehnis (2009), der in seiner Dissertation die Erziehungszeiten von Vätern untersuchte, stellte in seiner qualitativen Analyse fest, dass besonders das erste Lebensjahr des Kindes durch eine „hegemoniale Mütterlichkeit“ (ebd., S. 146) gekennzeichnet ist, in dem das „Idealbild der betreuenden Person“ (ebd., S. 147) der Frau bzw. Mutter zugeordnet ist. Ein Großteil der von ihm untersuchten Eltern verfolgte daher während der dreijährigen Erziehungszeit ein sequenzielles Modell: Im ersten Lebensjahr des Kindes blieben dabei die Mütter überwiegend vollständig zu Hause (wesentlich durch Stillzeiten begründet), während der Vater erst im zweiten Lebensjahr des Kindes zum Hauptbetreuer avancierte (ebd., S. 144ff.). Es ist daher zu bedenken, ob die Beschränkung des Elterngeldes auf das erste Lebensjahr des Kindes Väter möglicherweise systematisch ausschließt, bzw. tatsächlich lediglich auf die beiden Zusatzmonate (parallel zur Mutter) verweist.
Insgesamt wird, den Befunden der RWI-Studie zufolge, eine Übernahme von Elterngeldmonaten durch den Vater dann besonders wahrscheinlich, wenn die Eltern noch keine älteren Kinder haben, wenn die Mutter im Jahr vor der Geburt erwerbstätig war und der Vater einen besonders hohen Bildungsabschluss hat; auch Familien mit älteren Vätern beantragen häufiger Partnermonate als Familien mit jungen Vätern unter 25 Jahren (RWI 2009, S. 15). Auch ist der Anteil der Familien mit Partneranträgen bei Müttern mit geringer Ausbildung wesentlich niedriger, als dies bei Akademikerinnen der Fall ist (ebd., S. 20). Pfahl/Reuyß (2009, S. 96) kommen in ihrer Studie zum neuen Elterngeld überdies zu dem Schluss, dass das Elterngeld vor allem für solche Familien attraktiv ist, deren Einkommen ausreicht, um das Familienauskommen trotz reduzierter Einkünfte weiter zu sichern. An den Familien geht diese temporäre finanzielle Einschränkung trotzdem nicht vorbei: Fast die Hälfte der in der Studie befragten Männer gibt an, durch die Elternmonate einen bedeutenden finanziellen Verlust erlitten zu haben (ebd., S. 97).

\subsection{Bezugshöhe des Elterngeldes}

\subsubsection{Existenzsicherung für Mütter?}

Eine der Zielsetzungen des neuen Elterngeldes war es, eine eigenständige Existenzsicherung von Müttern und Vätern zu gewährleisten, die an eine Erwerbstätigkeit vor der Geburt des Kindes geknüpft ist. So sah die einkommensabhängige Elterngeldregelung in Deutschland bis Ende $2010^{\circ}$ vor, dass die Eltern im Elterngeldbezug $67 \%$ ihres ursprünglichen Nettogehalts bekommen. ${ }^{8}$

Zunächst ist daher die Frage interessant, für welchen Anteil der Eltern das Elterngeld tatsächlich einen (Teil-)Ersatz eines vormaligen Erwerbseinkommens ausmacht. So waren vor dem Elterngeldbezug 86,2 \% der Väter, aber nur 59,4 \% der Mütter erwerbstätig. Für 81,7 \% der Väter stellt das Elterngeld einen vollen Ersatz von Erwerbseinkommen dar; dies gilt jedoch nur für 36,9 \% der Mütter. Der Großteil der Mütter (44,0\%) hingegen bekommt nur den Mindestbetrag (16\% der Väter), $25 \%$ erhalten einen Geringverdienstzuschlag (8,1 \% der Väter) und für 5,5 \% der Mütter und für 13,2 \% der Väter stellt es eine Reduzierung des Einkommens dar. ${ }^{\circledR}$ Diese Zahlen verweisen auf die Langfristigkeit weiblicher, fa-

7 Die neue Regelung seit Anfang 2011 sieht nun vor, dass Eltern ab einem bereinigten Nettoeinkommen von mehr als $1.240 €$ monatlich nur noch $65 \%$ ihres ursprünglichen $\mathrm{Ge}$ halts als Elterngeld bekommen.

(8) Für erwerbslose Eltern, Schüler und Studenten und Hausfrauen wurde ein Mindestelterngeld in Höhe von $300 €$ bezahlt. Für Geringverdiener mit einem Nettoeinkommen von unter $1.000 €$ monatlich gab es einen Zuschlag, mit dem das Elterngeld bis zu $100 \%$ des alten Nettolohnes ausmachen konnte.

(9) Die Eltern sind hier weiter erwerbstätig, wenn auch in einem geringeren Umfang. 
TABELLE 2

Höhe des Elterngeldes bei Müttern und Vätern

Anteile in Prozent

\begin{tabular}{|c|c|c|c|c|c|c|c|c|c|}
\hline & $300 €$ & $300-500 €$ & $500-750 €$ & $750-1000 €$ & $1000-1250 €$ & $1250-1500 €$ & $1500-1800 €$ & $1800+€$ & Durchschnitt \\
\hline Mütter & 36,0 & 14,5 & 18,2 & 133 & 8,2 & 4,3 & 3,0 & 2,5 & $673 €$ \\
\hline Väter & 13,3 & 5,1 & 8,6 & 13,1 & 16,6 & 14,8 & 11,7 & 16,7 & $1147 €$ \\
\hline
\end{tabular}

TABELLE 3

Höhe des Elterngeldes von Müttern nach Anzahl der Kinder im Haushalt

Anteile in Prozent

Anzahl der Kinder im Haushalt

\begin{tabular}{|c|c|c|c|c|}
\hline Höhe des Elterngeldes $(€)$ im 1. Bezugsmonat & 1 & 2 & 3 & 4 und mehr \\
\hline 300 & 30,7 & 22,7 & 30,6 & 35,4 \\
\hline $300-500$ & 5,9 & 34,4 & 42,4 & 49,1 \\
\hline $500-750$ & 19,3 & 19,3 & 13,5 & 8,9 \\
\hline $750-1.000$ & 16,6 & 8,7 & 5,0 & 2,6 \\
\hline $1.000-1.250$ & 12,3 & 5,7 & 3,2 & 1,4 \\
\hline $1.250-1.500$ & 6,4 & 3,7 & 1,9 & 1,0 \\
\hline $1.500-1.800$ & 4,6 & 2,4 & 1,5 & 0,8 \\
\hline 1.800 und mehr & 4,3 & 3,1 & 1,9 & 0,8 \\
\hline
\end{tabular}

milienbedingter Berufsausstiege hin, die bei Vätern so nicht abbildbar sind. So geht aus der RWI-Studie 2009 hervor, dass zwar der überwiegende Anteil der Mütter, die ein erstes Kind bekamen, vor der Geburt erwerbstätig war (74 \%), dass jedoch der Anteil an Erwerbstätigen mit der Geburtenfolge deutlich abnimmt: $43 \%$ der zweifachen Mütter, nur $31 \%$ der dreifachen Mütter und $13 \%$ der Mütter eines vierten oder weiteren Kindes waren vor der Geburt erwerbstätig. Auch der Umfang der vormaligen Erwerbstätigkeit nimmt mit der Kinderfolge deutlich ab (RWI 2009, S. 43). Für die Väter zeigen sich solche Zusammenhänge nicht. Damit - und unter Berücksichtigung des nach wie vor existierenden Lohndifferenzials zwischen den Geschlechtern - unterscheidet sich erwartungsgemäß auch die Höhe des Elterngeldes (Tabelle 2).

Die größte Gruppe der Mütter (36 \%) bekommt ein monatliches Elterngeld in Höhe von $300 €$. Nach den Befunden der RWI-Studie handelt es sich bei diesen Frauen überwiegend um Hausfrauen ohne eigenes Einkommen, um Studentinnen/Schülerinnen, um arbeitslose Frauen sowie um geringfügig und/oder temporär beschäftigte Erwerbstätige (RWI 2009, S. 28). Die Hälfte der Mütter bleibt in den Einkommensgruppen unter $500 €$; im Schnitt beträgt das El- terngeld der Mütter daher auch nur $673 €$ monatlich. Die Hälfte der Väter bekommt bis zu $1.250 €$ monatlich, im Durchschnitt $1.147 €$. Mit 16,7 \% sind jedoch auch einige Väter in der obersten Elterngeldgruppe von $1.800 €$ und mehr monatlich zu finden.

\subsubsection{Väter bekommen mehr, aber das kürzer}

Interessanterweise zeigen die Daten auch, dass die Väter mit höherem Elterngeldanspruch tendenziell weniger Elternmonate in Anspruch nehmen: Die Väter mit $300 €$ Elterngeld beziehen im Schnitt 4,5 Monate Elterngeld, die Väter mit $1.800 €$ Elterngeld hingegen nur 2,7 Monate. Bei den Müttern zeigen sich keine entsprechenden Effekte. Hingegen wirken sich auf die Höhe des Elterngeldes vorherige Geburten deutlich aus: So handelt es sich bei insgesamt 45,2 \% der Elterngeld beziehenden Mütter nicht um das erste, sondern um das zweite $(32,1 \%)$ oder das dritte oder weitere Kind (14,1\%). Das heißt, ein Großteil der Mütter geht bereits nicht aus einer lückenlosen Vollzeiterwerbstätigkeit heraus in die Elterngeldphase. Der Anteil der Mütter, welche sich in den geringeren Elterngeldgruppen befinden, steigt daher auch mit der Anzahl der Kinder sehr deutlich an (Tabelle 3); bei den Vätern zeigt sich ein solcher Zusammenhang wiederum nicht (vgl. hierzu auch RWI 2009, S. 24).

\section{Das Elterngeld im Licht der Wahlfreiheit}

Die Intention des Elterngeldes ist es, einen „Schonraum“ (BMFSFJ 2010) für Eltern im ersten Lebensjahr des Kindes zu schaffen und wegfallendes Erwerbseinkommen zu ersetzen - aber eben nur Erwerbseinkommen und dies genau genommen nur für diejenigen, für die auch zwei Drittel ihres Einkommens zum Leben reichen. ${ }^{\circledR}$ Daher wurde die

(10) Der Begriff des "Schonraumes" ist zudem eine neuere Deutung des Elterngeldes. Zuvor wurde der kurze Geburtenanstieg nach Einführung des Elterngeldes auch als Erfolg gedeutet. 
Leistung als Lohnersatzleistung ausgestaltet. Das Elterngeld sollte zwei Drittel des bisherigen Nettogehaltes ersetzen, und damit eine Existenzsicherung ermöglichen. Die Daten zeigen, dass dies nur für einen ausgewählten Anteil der Elterngeldbezieher zutrifft. Es greift der Matthäus-Effekt: „Wer hat, dem wird gegeben.“ (Wimbauer et al. 2008, S. 20)

Insbesondere Frauen liegen zu hohen Anteilen mit dem Elterngeld unterhalb der Armutsrisikogrenze. Vor dem Hintergrund, dass der überwiegende Anteil der atypisch, im Niedriglohnsektor oder anderweitig prekär beschäftigten Personen weiblich ist (vgl. Kalina/Weinkopf 2010, S. 5f.), überrascht es nicht, dass eine lohnabhängige Leistung für Frauen im Vergleich zu Männern geringer ausfällt. Bezieht man ein, dass Mütter in Deutschland im europäischen Vergleich bereits beim ersten Kind überdurchschnittlich lange Erwerbsausstiege haben, beim zweiten und dritten Kind häufig nur in Teilzeit in den Beruf zurückkehren, dann überrascht noch weniger, dass der Großteil der Mütter nicht vom Elterngeld in Existenz sichernder Weise profitiert ( $\mathrm{Ta}$ belle 3; vgl. auch BMFSFJ 2011).

\subsection{Frauen und Wahlfreiheit unter ungleichen Bedingungen}

Die anvisierte Wahlfreiheit einer im ersten Jahr abgesicherten Existenz kann für diese Frauen nicht angenommen werden. Nicht zuletzt führt die normative Kraft geschlechtsspezifischer Erwartungen an die Ausgestaltung von Elternschaft trotz der geringeren Beträge zu Erwerbsausstiegen von Frauen. In einer zweiten Dimension führt das Elterngeld zu einer Erhöhung der Ungleichheit unter Frauen (vgl. Martinek 2010). Prekär beschäftigte Frauen, Alleinerziehende, nicht erwerbstätige Frauen und ein Anteil der Migrantinnen können nicht vom Elterngeld profitieren, weil sie entweder keine Leistungsberechtigung haben oder nicht ausreichend im Erwerbsleben Fuß fassen. Für Alleinerziehende gilt zudem, dass der Bezug der Partnermonate von ihnen nur dann realisiert werden kann, wenn sie das alleinige Sorgerecht für das Kind innehaben oder eine entsprechende Anordnung bezüglich des Aufenthaltsbestimmungsrechts.

Vor dem Hintergrund der oben entwickelten drei Entscheidungsdimensionen, die durch das Elterngeld befördert werden sollen - Anstieg der Geburtenrate, Verkürzung der Erwerbsunterbrechungen von Frauen und Beteiligung der Väter an der Erziehungszeit - lassen sich drei Aspekte der Wahlfreiheit im Sinne autonomer Entscheidungen ableiten.

\subsection{Elterngeld und Geburtenzahlen}

Kinderlosigkeit ist ein Phänomen, das auf unterschiedlichen Entscheidungen oder Motivlagen basieren kann (vgl. Rost/ Schneider 1996; Tölke/Diewald 2003). Das Elterngeld hat nicht zu einem Geburtenanstieg geführt. Vor dem Hintergrund, dass eine Entscheidung für ein Kind in der Regel einen über zwölf bzw. 14 Monate hinausreichenden Zeit- raum umfasst, ist dies nicht verwunderlich. Gleichwohl wäre es wünschenswert, Entscheidungsprozesse für oder gegen ein Kind vor dem Hintergrund des Elterngeldes zu beforschen, was gesonderte Auswertungen erfordern würde. In Richtung der langfristigen Erwerbsperspektive legen die Elterngelddaten nahe, dass zumindest Mütter sowohl starken wirtschaftlichen Restriktionen unterliegen als auch längere Erwerbsausstiege als Männer haben. Hier ist wenigstens zu vermuten, dass Frauen mit diesen Perspektiven sich nicht ohne Zögern für ein weiteres Kind entscheiden werden.

\subsection{Elterngeld und Dauer der Erwerbsausstiege}

Mehr als 90 \% der Mütter nahmen zehn bis zwölf Monate Elterngeld in Anspruch. Es wird aus den Daten nicht deutlich, ob diese Mütter direkt wieder in den Beruf einsteigen. Die Zahlen zur Müttererwerbstätigkeit deuten zumindest an, dass sich die Geburt eines Kindes noch immer negativ auf die Erwerbsbiografie auswirkt. Die RWI-Studie legt nahe, dass einerseits diejenigen, die unter anderen Umständen kürzer als zwölf Monate in Elternzeit geblieben wären, zu längeren Erwerbsausstiegen angeregt werden, andererseits scheint sich die Dauer der Erwerbsausstiege insgesamt zu verkürzen. Hierbei ist allerdings zu beachten, dass in den RWI-Daten die Wiedereinstiegswünsche abgebildet wurden, nicht die tatsächlichen Wiedereinstiege.

Die kurze Übernahme der Elternzeit, verbunden mit einem kurzfristigen Erwerbsausstieg und dann gegebenenfalls einem Wiedereinstieg in Teilzeit, stellt anscheinend auch für Mütter die legitime Wahl dar. Wie McRobbie (2009) in der Untersuchung postfeministischer diskursiver Phänomene darstellt, sind auch Frauen vielfältigen Normierungen von Mutterschaft unterworfen, die sich längst nicht mehr ausschließlich in einem Leben als Hausfrau und Mutter erschöpfen. Hier greifen finanzielle Restriktionen und normative Leitbilder ineinander und, so die Annahme, ergänzen sich gegenseitig zum vorliegenden Modell der „kurzen" Elternzeit für Väter und Mütter.

Bei Frauen, die ihr zweites, drittes oder viertes Kind bekommen, schränkt sich die Wahlfreiheit noch mehr ein. Sie sind zu deutlich geringeren Anteilen vor der Geburt erwerbstätig und dies häufig nur in Teilzeit. Sofern keine Erwerbstätigkeit vorliegt, besteht nur Anspruch auf den Mindestbetrag, der im Vergleich zum vorherigen Erziehungsgeld eine Halbierung der Leistung darstellt. Erwerbsanreize werden dadurch für diese Gruppe höchstwahrscheinlich nicht entstehen.

\subsection{Mehr involvierte Väter?}

Der Anteil der Väter, der grundsätzlich Elternzeit nimmt, steigt, seit es das Elterngeld gibt, enorm an. Er liegt bei etwa einem Fünftel und der überwiegende Teil nimmt zwei Monate Elternzeit. Das deutet darauf hin, dass die sogenannten Partnermonate tatsächlich in Anspruch genommen werden. 
Väter geben gemäß den RWI-Daten an, dass sie aus finanziellen Gründen ihre Arbeitszeit nicht reduzieren könnten. Ein kleiner Anteil von Vätern erhält ein Elterngeld in einer Höhe, die eine Existenzsicherung jenseits der Armutsrisikogrenze ermöglicht. Dennoch nehmen diese Väter meist nur kurz, überwiegend für zwei Monate, Elternzeit. Zurzeit stehen keine paarbezogenen Daten zur Verfügung, sodass nicht festgestellt werden kann, wie das Einkommen der jeweiligen Mütter, sprich der Partnerinnen der Väter in Elternzeit, ausfällt.

Da Unterschiede in der Elterngeldhöhe bei ersten, zweiten oder dritten Kindern für Väter nicht zu finden sind, lässt sich aus den vorliegenden Daten nicht beantworten, ob das Elterngeld einen Einfluss auf die Dauer des Elterngeldbezuges hat. Das vorliegende Material deutet darauf hin, dass die Strukturbedingungen des Arbeitsmarktes mindestens subjektiv für Väter ohnehin nur kurze Erwerbsausstiege ermöglichen. Diese scheinen durch das Elterngeld zwar in greifbare Nähe zu rücken, jedoch nur ab einer höheren Einkommensstufe.

Engagierte Vaterschaft, welche auch die Unterbrechung oder die Reduktion von Erwerbstätigkeit beinhaltet - und damit auch egalitäre Aufteilung der Kinderbetreuung scheint demnach derzeit nur dann realisierbar zu sein, wenn die Familie ausreichend finanziell abgesichert ist oder die Mutter ein ähnlich hohes Einkommen und eine stabile berufliche Position hat. Hinzu kommt, dass engagierte Vaterschaft zwar ein politisches Leitbild ist, jedoch anderen Normen von Männlichkeit, wie hohem beruflichen Engagement, entgegensteht. Auf der ideellen Ebene ist für Väter daher kaum eine Entscheidungsautonomie im Hinblick auf eine Elternzeit, die über den finanziellen Anreiz der zwei Monate hinausgeht, anzunehmen, zumal dann auf sozialer bzw. der Ebene des Geschlechterverhältnisses ein höherer Legitimierungsdruck entsteht. Kurzum: Die unterstellten Mitnahmeeffekte, wegen derer Väter zwei Monate Elternzeit in Anspruch nehmen, können umgekehrt als Interpretationsoption für Väter betrachtet werden, Elternzeit zu nehmen, ohne in den Verdacht eines zu familienorientierten Verhaltens zu geraten. Exemplarisch kann hier Connells (2010) Studie zum „gläsernen Turm“ angeführt werden, wo ähnliche Mechanismen in einer neoliberal geprägten $\mathrm{Ar}$ beitswelt beschrieben werden.

\section{Fazit: Perspektiven für die Elterngeldpolitik}

Die vorliegenden Daten belegen, dass das Elterngeld nicht für alle Familien zu einem Gewinn an Autonomie und damit Wahlfreiheit geführt hat. Im Gegenteil, ärmere Paare erleben im Vergleich zum Erziehungsgeld eine deutliche Einschränkung ihrer finanziellen Handlungsspielräume. Hinsichtlich der ideellen Anerkennung lässt sich tendenzi- ell feststellen, dass das Elternwerden als solches zu einer bedeutsamen gesellschaftlichen Aufgabe avanciert ist, die nicht nur für Mütter zu Anerkennung führt. Dabei werden bestimmte Lebensmodelle sowohl durch die finanzielle Förderung als auch die ideelle Engführung besonders gefördert: die heterosexuelle Paarfamilie mit zwei erwerbstätigen Erwachsenen, in der in der Regel der Mann mehr als die Frau verdient und sich dennoch in begrenztem, die Erwerbstätigkeit nicht über Gebühr einschränkendem Ausmaß an Familienaufgaben beteiligt.

Mit der Umsetzung des Sparpakets (1. Januar 2011) hat sich die Struktur des Elterngeldes maßgeblich verändert. Während der sozialpolitische Ansatz noch deutlicher in den Hintergrund gerückt ist, wurde auch die Ausgestaltung des Lohnersatzes für Einkommen zwischen 1.240 und $1.800 €$ weniger attraktiv. Das Elterngeld in seiner ursprünglichen Form ist damit nur noch für den Korridor zwischen einem Nettoeinkommen von 1.000 und $1.240 €$ erhalten. Fraglich ist auf der einen Seite, wie sich dies auf die Partizipation von Vätern an Elternzeit auswirkt. Da bereits heute viele Väter aus finanziellen Gründen keine Möglichkeit sehen, auch nur kurzfristig aus dem Beruf auszusteigen oder die Arbeitszeit zu reduzieren, wird sich diese Haltung womöglich verstärken. Für die gleichberechtigte Aufteilung von Familienaufgaben wäre dies ein Rückschritt. Die Gruppe der Väter, die bisher eine Vorreiterrolle in der Beteiligung an der Elternzeit innehatten, die mithin die zwei Partnermonate in Anspruch genommen haben und auch für Kollegen eine Vorbildfunktion hatten, könnte dadurch wieder schrumpfen. Dies wäre angesichts der bislang erfreulichen Entwicklung des Väteranteils beim Elterngeld höchst bedauerlich.

Familien im SGB-II-Bezug erhalten heute weder Elterngeld noch Erziehungsgeld. Im Vergleich zur Situation vor 2007 ist dies ein Rückschritt. Binnen weniger als vier Jahre wurde für diese Eltern eine Leistung vollständig gestrichen. Die dahinter stehende Logik, eine Elternschaft womöglich erst einzugehen, wenn eine Erwerbstätigkeit vorliegt, könnte in diesen Familien massive Konsequenzen für die Familienplanung haben. Vor dem Hintergrund, dass der Berufseinstieg auch für Akademiker heute kaum ohne Hürden verläuft, das heißt von befristeter Beschäftigung, hohen Mobilitätsanforderungen, Teilzeitbeschäftigung und mitunter schlecht entlohnten Praktika geprägt ist, stellt die Einschränkung des Elterngeldes auch für diese Gruppe ein Hindernis dar. Kinderwünsche werden dadurch womöglich aufgeschoben und generell ist es möglich, dass die zuletzt vergleichsweise stabile Geburtenentwicklung wieder einen rückläufigen Trend annimmt.

Gegenüber einer ursprünglich geplanten Ausweitung der Partnermonate und womöglich auch einer Einführung einer bezahlten Pflegezeit, geschweige denn der im Siebten Familienbericht der Bundesregierung (BMFSFJ 2005) empfohlenen Einführung von Optionszeiten, scheint das Projekt Elterngeld sich sukzessive zurück zu entwickeln.

Im Sinne einer Ermöglichung von Handlungsautonomie für alle Familien wären folgende Aspekte als Perspek- 
tive des Elterngeldes denkbar: ein Existenz sichernder Sockelbetrag, Individualansprüche für Mütter und Väter sowie ein höherer Lohnersatz als in der bisherigen Fassung. Angesichts der Kürzungen und der neuen Debatte zur „Wirksamkeit“ des Elterngeldes, wie sie 2011 von der Bundes-FDP angefacht wurde, scheint jedoch eine Streichung des Elterngeldes zumindest nicht mehr tabuisiert zu sein. Dem stehen die dargestellten Überlegungen zur „Wirkung“ des Elterngeldes durchaus entgegen, wenngleich ihr kritischer Gehalt ausreichend Anlass gibt, Politikentwicklung zu betreiben.

\section{LITERATUR}

Auth, D. (2007): Pronatalistischer Aktionismus: von der bevölkerungspolitischen Instrumentalisierung und Ökonomisierung der Familienpolitik in Deutschland, in: Auth, D./Holland-Cunz, B. (Hrsg.): Grenzen der Bevölkerungspolitik. Strategien und Diskurse demographischer Steuerung, Leverkusen/Opladen, S. 81-102

Beck, U. (1986): Risikogesellschaft. Auf dem Weg in eine andere Moderne, Frankfurt a. M.

Bothfeld, S. (2005): Vom Erziehungsurlaub zur Elternzeit, Frankfurt a. M. Bühler-Niederberger, D. (2005): Kindheit und die Ordnung der Verhältnisse. Von der gesellschaftlichen Macht der Unschuld und dem kreativen Individuum, Weinheim/Basel

Bundesministerium für Familie, Senioren, Frauen und Jugend (BMFSFJ) (2005): Siebter Familienbericht der Bundesregierung, Berlin Bundesministerium für Familie, Senioren, Frauen und Jugend (BMFSFJ) (2006): Einkommensersatz Elterngeld: 365.000 Familien in Deutschland profitieren, Pressemitteilung vom 11. 05., http://www.bmfsfj.de/BMFSFJ/Service/ Archiv/16-Legislaturperiode/pressemitteilungen,did=75678.html (letzter Zugriff: 04. 03. 2011)

Bundesministerium für Familie, Senioren, Frauen und Jugend (BMFSFJ) (2010): Das Elterngeld. Beitrag auf der Homepage des BMFSFJ vom 17. 12., www.bmfsfj.de (letzter Zugriff: 04. 03. 2011)

Bundesministerium für Familie, Senioren, Frauen und Jugend (BMFSFJ) (Hrsg.) (2011): Zeit für Familie. AusgewählteThemen des 8. Familienberichts, Berlin Connell, R. (2006): Der gemachte Mann. Konstruktion und Krise von Männlichkeiten, Wiesbaden

Connell, R. (2010): Im Inneren des gläsernen Turms, in: Feministische Studien 28 (1), Hannover, S. 8-24

Ehnis, P. (2009): Väter und Erziehungszeiten. Politische, kulturelle und subjektive Bedingungen für mehr Engagement in der Familie, Sulzbach

Fraser, N. (1994): Feminist Contentions: A Philosophical Exchange, New York Fthenakis, W. E./Minsel B. (2002): Die Rolle des Vaters in der Familie, Stuttgart et al.

Jurczyk, K./Keddi, B./Lange, A./Zerle, C. (2009): Zur Herstellung von Familie. Wie sich die Alltagspraxis von Vätern, Müttern und Kindern erforschen lässt ein Werkstattbericht, Deutsches Jugendinstitut e.V., Bulletin Plus, DJI, Bulletin 4 (88), München

Jurczyk, K./Schier, M./Szymenderski, P./Lange, A./Voß, G. G. (2009): Entgrenzung von Arbeit - Entgrenzung von Familie. Grenzmanagement im Alltag als neue Herausforderung, Berlin

Jurzcyk, K./Schutter, S. (2012): Familienpolitik, in: Kreft, D./Mielenz, I. (Hrsg.): Wörterbuch Soziale Arbeit, Weinheim (im Erscheinen)
Kalina T./Weinkopf C. (2010): Niedriglohnbeschäftigung 2008: Stagnation auf hohem Niveau - Lohnspektrum franst nach unten aus, IAQ-Report (06), Duis burg/Essen, http://www.iaq.uni-due.de/iaq-report/2010/report2010-06.pdf Martinek, H. (2010): Die Einführung des Elterngeldes: Ermöglichung der finan ziellen Unabhängigkeit für (alle) Frauen?, in: Auth, D./Buchholz, E./Janczyk, S. (Hrsg): Selektive Emanzipation, Opladen, S. 151-172

McRobbie, A. (2009): The Aftermath of Feminism. Gender, Culture, and Social Change, London

Pfahl, S./Reuyß, S. (2009): Das neue Elterngeld. Erfahrungen und betriebliche Nutzungsbedingungen von Vätern. Eine explorative Studie, Düsseldorf Rheinisch-Westfälisches Institut für Wirtschaftsforschung (RWI) (2009): Evaluation des Gesetzes zum Elterngeld und zur Elternzeit - Studie zu den Auswirkungen des BEEG auf die Erwerbstätigkeit und die Vereinbarkeitsplanung, Endbericht, Essen

Rost, H./Schneider, N. F. (1996): Gewollt kinderlose Ehen, in: Buba, H.-P./ Schneider, N. F. (Hrsg.): Familie zwischen gesellschaftlicher Prägung und individuellem Design, Opladen, S. 245-259

Saxonberg, S. (2009): Freedom of Choice through the Promotion of Gender Equality, in: Social Policy \& Administration 43 (6), S. 666-679

Schutter, S. (2005): Demografie als Argument für eine "nachhaltige Familienpolitik“?, in: Informationen für Einelternfamilien (03), S. 1-4

Schutter, S. (2011): "Richtige" Kinder: Von heimlichen und folgenlosen Vaterschaftstests, Wiesbaden

Statistisches Bundesamt (2011): Statistik zum Elterngeld. Gemeldete beendete Leistungsbezüge, 3. Vierteljahr 2011, http://www.destatis.de/jetspeed/portal/ $\mathrm{cms} /$ Sites/destatis/Internet/DE/Content/Publikationen/Fachveroeffentlichungen /Sozialleistungen/Elterngeld/ElterngeldGemeldeteBeendeteBezuegeVj,

templateld=renderPrint.psml

Tölke, A./Diewald, M. (2003): Berufsbiografische Unsicherheiten und der Über gang zur Elternschaft bei Männern, in: Bien, W./Marbach, J. H. (Hrsg.): Partnerschaft und Familiengründung. Ergebnisse der dritten Welle des Familien-Survey, Opladen, S. 349-384

Wimbauer, C./Henninger, A./Dombrowski, R. (2008): Wer hat, dem wird gegeben. Vom neuen Elterngeld profitieren vor allem die Besserverdienenden, in: WZB-Mitteilungen (120), S. 20-22

Wirth, H./Schutter, S. (2011): Versorger und Verlierer, in: DJI Impulse 92/93 (1), S. $5-8$

\section{AUTORINNEN}

SABINA SCHUTTER, Dr., ist Grundsatzreferentin für Familienpolitik am Deutschen Jugendinstitut (DJI) e.V. in München. Arbeitsschwerpunkte: Familienpolitik und familienbezogene Leistungen, soziale Ungleichheit und Armut von Familien.

schutter@dji.de

CLAUDIA ZERLE-ELSÄßER ist wissenschaftliche Mitarbeiterin in der Abteilung Familie und Familienpolitik des Deutschen Jugendinstituts e.V. in München. Arbeitsschwerpunkte: Quantitative Familiensoziologie, Vaterschaft, Familiengründung, Alltag von Männern, Frauen und Kindern.

zerle@dji.de 